\title{
Suicide among children and adolescents in Canada: trends and sex differences, 1980-2008
}

\author{
Robin Skinner MSP, Steven McFaull MSc
}

See related commentary by Kirmayer on page 1015 and at www.cmaj.ca/lookup/doi/10.1503/cmaj.120509

\begin{abstract}
Background: Suicide is the second leading cause of death for young Canadians (1019 years of age) - a disturbing trend that has shown little improvement in recent years. Our objective was to examine suicide trends among Canadian children and adolescents.

Methods: We conducted a retrospective analysis of standardized suicide rates using Statistics Canada mortality data for the period spanning from 1980 to 2008 . We analyzed the data by sex and by suicide method over time for two age groups: 10-14 year olds (children) and 15-19 year olds (adolescents). We quantified annual trends by calculating the average annual percent change (AAPC).
\end{abstract}

Results: We found an average annual decrease of $1.0 \%$ ( $95 \%$ confidence interval
[CI] -1.5 to -0.4 ) in the suicide rate for children and adolescents, but stratification by age and sex showed significant variation. We saw an increase in suicide by suffocation among female children (AAPC $=8.1 \%, 95 \% \mathrm{Cl} 6.0$ to 10.4 ) and adolescents (AAPC $=8.0 \%, 95 \% \mathrm{Cl}$ 6.2 to 9.8 ). In addition, we noted a decrease in suicides involving poisoning and firearms during the study period.

Interpretation: Our results show that suicide rates in Canada are increasing among female children and adolescents and decreasing among male children and adolescents. Limiting access to lethal means has some potential to mitigate risk. However, suffocation, which has become the predominant method for committing suicide for these age groups, is not amenable to this type of primary prevention.
$\mathrm{S}$ uicide was ranked as the second leading cause of death among Canadians aged $10-34$ years in $2008 .{ }^{1}$ It is recognized that suicidal behaviour and ideation is an important public health issue among children and adolescents; disturbingly, suicide is a leading cause of Canadian childhood mortality (i.e., among youths aged $10-19$ years)., ${ }^{2,3}$

Between 1980 and 2008, there were substantial improvements in mortality attributable to unintentional injury among 10-19 year olds, with rates decreasing from 37.7 per 100000 to 10.7 per 100 000; suicide rates, however, showed less improvement, with only a small reduction during the same period (from 6.2 per 100000 in 1980 to 5.2 per 100000 in 2008). ${ }^{1}$

Previous studies that looked at suicides among Canadian adolescents and young adults (i.e., people aged 15-25 years) have reported rates as being generally stable over time, but with a marked increase in suicides by suffocation and a decrease in those involving firearms. ${ }^{2}$ There is limited literature on self-inflicted injuries among children 10-14 years of age in
Canada and the United States, but there appears to be a trend toward younger children starting to self-harm. ${ }^{3,4}$ Furthermore, the trend of suicide by suffocation moving to younger ages may be partly due to cases of the "choking game" (selfstrangulation without intent to cause permanent harm) that have been misclassified as suicides..$^{5-7}$

Risk factors for suicidal behaviour and ideation in young people include a psychiatric diagnosis (e.g., depression), substance abuse, past suicidal behaviour, family factors and other life stressors (e.g., relationships, bullying) that have complex interactions. ${ }^{8}$ A suicide attempt involves specific intent, plans and availability of lethal means, such as firearms, ${ }^{9}$ elevated structures ${ }^{10}$ or substances. ${ }^{11}$ The existence of "pro-suicide" sites on the Internet and in social media ${ }^{12}$ may further increase risk by providing details of various ways to commit suicide, as well as evaluations ranking these methods by effectiveness, amount of pain involved and length of time to produce death. ${ }^{13-15}$

Our primary objective was to present the patterns of suicide among children and adolescents (aged 10-19 years) in Canada.
Competing interests: None declared.

This article has been peer reviewed.

Correspondence to: Robin Skinner,

robin.skinner@phac-aspc .gc.ca

CMAJ 2012. DOI:10.1503 /cmaj.111867 


\section{Methods}

We accessed Statistics Canada mortality data from the Public Health Agency of Canada. For each death occurring before 2000 , the external cause of injury or poisoning was reported by the provincial or territorial Vital Statistics Registry and classified according to the International Classification of Diseases, 9th Revision (Clinical Modification) (ICD-9-CM); the International Statistical Classification of Diseases and Related Health Problems, 10th revision (ICD-10) was used to classify the causes of deaths occurring thereafter. Comparability studies describing the implementation of the ICD-10 in 2000 to understand trends in mortality between the ICD-9-CM and ICD-10 concluded that there was no effect on the continuity of suicide data. ${ }^{16,17}$

We extracted deaths by suicide based on ICD-9-CM and ICD-10 codes $^{18}$ (X60-X84, Y87.0), and by the primary means of suicide seen among children and adolescents (hanging, strangling, suffocation [X70, henceforth referred to as "suffocation"]; poisoning [X60-X69]; and firearms [X72-X74]) for the period 1980-2008. We categorized the data into two age groups (children [10-14 years] and adolescents [15-19

\begin{tabular}{|c|c|c|c|c|c|c|c|c|}
\hline \multirow[b]{3}{*}{ Year } & \multicolumn{8}{|c|}{ Method of suicide, no. } \\
\hline & \multicolumn{2}{|c|}{ All } & \multicolumn{2}{|c|}{ Firearm } & \multicolumn{2}{|c|}{ Poisoning } & \multicolumn{2}{|c|}{ Suffocation } \\
\hline & Male & Female & Male & Female & Male & Female & Male & Female \\
\hline 1980 & 249 & 50 & 129 & 11 & 39 & 21 & 64 & 10 \\
\hline 1981 & 275 & 52 & 153 & 20 & 19 & 16 & 85 & 12 \\
\hline 1982 & 270 & 39 & 128 & 8 & 37 & 17 & 80 & 5 \\
\hline 1983 & 270 & 42 & 151 & 10 & 27 & 12 & 83 & 16 \\
\hline 1984 & 244 & 36 & 106 & 7 & 27 & 17 & 95 & 6 \\
\hline 1985 & 198 & 40 & 93 & 13 & 29 & 11 & 61 & 12 \\
\hline 1986 & 218 & 47 & 98 & 15 & 33 & 12 & 75 & 14 \\
\hline 1987 & 226 & 48 & 105 & 11 & 27 & 16 & 81 & 17 \\
\hline 1988 & 235 & 34 & 103 & 6 & 23 & 11 & 94 & 13 \\
\hline 1989 & 237 & 35 & 105 & 6 & 30 & 9 & 85 & 16 \\
\hline 1990 & 205 & 49 & 100 & 8 & 21 & 19 & 72 & 18 \\
\hline 1991 & 236 & 45 & 107 & 7 & 16 & 15 & 96 & 18 \\
\hline 1992 & 224 & 59 & 100 & 11 & 17 & 15 & 93 & 29 \\
\hline 1993 & 219 & 62 & 95 & 13 & 17 & 10 & 96 & 34 \\
\hline 1994 & 242 & 59 & 89 & 5 & 18 & 11 & 115 & 37 \\
\hline 1995 & 239 & 68 & 66 & 8 & 19 & 7 & 121 & 41 \\
\hline 1996 & 221 & 50 & 58 & 8 & 18 & 10 & 120 & 31 \\
\hline 1997 & 255 & 68 & 71 & 7 & 18 & - & 147 & 54 \\
\hline 1998 & 222 & 79 & 60 & 7 & 22 & 11 & 120 & 56 \\
\hline 1999 & 230 & 55 & 61 & 6 & 13 & - & 139 & 42 \\
\hline 2000 & 202 & 69 & 42 & 7 & 20 & - & 125 & 53 \\
\hline 2001 & 170 & 64 & 28 & - & 13 & 12 & 109 & 46 \\
\hline 2002 & 179 & 71 & 47 & - & 13 & - & 102 & 55 \\
\hline 2003 & 180 & 63 & 28 & 5 & 9 & 7 & 129 & 47 \\
\hline 2004 & 178 & 60 & 29 & - & 9 & 7 & 131 & 44 \\
\hline 2005 & 165 & 91 & 29 & - & 8 & 5 & 114 & 79 \\
\hline 2006 & 125 & 58 & 24 & - & 8 & 5 & 80 & 48 \\
\hline 2007 & 149 & 69 & 29 & - & 8 & 5 & 92 & 59 \\
\hline 2008 & 156 & 77 & 22 & - & 10 & 10 & 116 & 61 \\
\hline
\end{tabular}


years]), by sex and by method of suicide. Mortality was standardized to the population of Canada in 1991.

\section{Statistical analysis}

We examined temporal trends for the period 1980-2008 and for the ages 10-19 years using the average annual percent change (AAPC) in suicide rate per 100000 population. The AAPC was calculated (with $95 \%$ confidence intervals [CI]) separately for sex, age and method of suicide using the equation

$$
\mathrm{AAPC}=\left[e^{\beta}-1\right] \times 100
$$

where $\beta$ is the slope from a regression of $\log$ rates on year. We used the Canadian population in 1991 as the standard population for computing age-standardized rates. We smoothed year-toyear variations, likely the result of small sample sizes, by applying a 5-point central moving average to these rates.

\section{Results}

There were 233 suicides among young Canadians in 2008, accounting for $20.4 \%$ of all deaths for people aged 10-19 years. In comparison, suicides comprised $1.5 \%$ of all deaths in Canada during the same year. The suicide rate among Canadians aged 10-19 years showed a significant annual average decrease of $1.0 \%$ ( $95 \% \mathrm{CI}-1.5$ to -0.4 ) during the study period (1980-2008); however, stratification by age and sex showed significant variation. Table 1 provides the numbers of suicides among 1019 year olds, by sex and method, during the study period.

In 2008, the suicide rate among children aged 10-14 years was 1.2 per $100000(n=25$, $64 \%$ male), accounting for $10 \%$ of all deaths in this age group, $88 \%$ of which were the result of suffocation ( $n=22,64 \%$ male). Among adolescents aged 15-19 years, the overall suicide rate in 2008 was 9.2 per $100000(n=208,67 \%$ male), accounting for $23 \%$ of all deaths, with suffocation also the primary means $(73 \%$ of suicides in boys [ $n=102]$ and $78 \%$ of suicides in girls $[n=53])$.

We restricted further analyses to the primary methods of suicide - suffocation, firearms and poisoning. These methods accounted for almost $95 \%$ of suicides among people aged 10-19 years in 2008 .

Suicide rates among boys aged 10-14 years showed no significant change during the 29-year period of the study (AAPC $=-0.9 \%[95 \%$ $\mathrm{CI}-2.2$ to 0.4$]$ ) (Figure 1 ). The overall rate was
1.6 per 100000 in 2008, and suffocation was the primary method (data not shown). None of the trends were statistically significant, with the exception of the decline in the use of firearms.

Suicide rates among male adolescents (aged 15-19 years) showed a general downward trend (Figure 2), from 19.0 per 100000 in 1980 to 12.1 per 100000 in $2008(\mathrm{AAPC}=-2.0 \%$, $95 \%$ CI -2.6 to -1.4$)$. Suicides involving firearms began to decline in 1992, showing an average annual decrease of $6.7 \%$ (Figure 1), whereas suicides by suffocation increased annually by an average of $1.8 \%$. Suffocation overtook firearms as the leading method of suicide for this group in 1994 (Table 2). Poisonings also decreased to a rate of 0.8 per 100000 in 2008 (data not shown).

Among girls aged 10-14 years, suicide rates increased sporadically from 0.6 per 100000 in 1980 to 0.9 per 100000 in 2008. Figure 3 shows the trend for this group. During the study period, deaths by suffocation increased (AAPC $=8.1 \%$, 95\% CI 6.0 to 10.4) (Figure 1), whereas deaths by poisoning decreased by an average of $2.9 \%$ per year. No significant changes were seen for suicides involving firearms for this group during this period (data not shown).

Among female adolescents (aged 1519 years), suicide rates increased during the study period, from 3.7 per 100000 to 6.2 per 100000 (AAPC $=1.8 \%, 95 \%$ CI 1.0 to 2.5 ) (Figure 1). Of note are the shifting patterns in the primary methods of suicide used by this group (Table 2). These patterns are similar to those

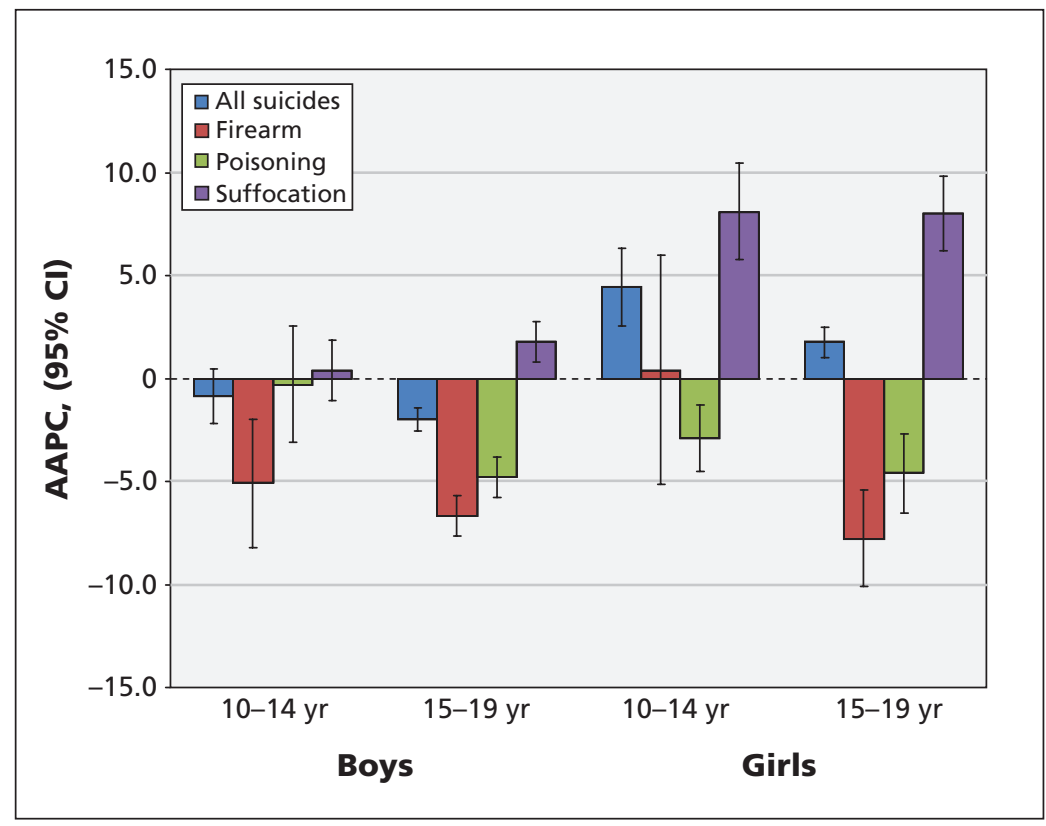

Figure 1: Suicides among Canadian children and adolescents, 1980-2008, by age group, sex and method. AAPC $=$ average annual percent change, $\mathrm{Cl}=$ confidence interval. 
seen in girls aged 10-14 years, with a steady average annual increase in suicides by suffocation of $8.0 \%$ (Figure 1). Suicides involving firearms and poisoning both decreased significantly; use of firearms decreased by an average of $7.8 \%$ per year, and poisoning decreased by an average of $4.6 \%$ per year (Figure 1).

\section{Interpretation}

Between 1980 and 2008, although the rates of unintentional injuries among children and adolescents have decreased substantially, suicide rates have shown much less change. Among female children and adolescents, overall suicide rates have increased, with suffocation becoming the most common method used. For the same group, suicides involving firearms and poisonings have decreased.

During the study period (1980-2008), the overall suicide rate among male adolescents (aged 15-19 years) underwent a statistically significant, although not large, decline; we also saw an increase in suicides by suffocation and a decrease in suicides involving firearms for this group.

Suffocation has become the leading method of suicide among children and adolescents, regardless of sex. A qualitative study by Biddle and coworkers examined factors that influenced people to select hanging to attempt suicide. Participants reported that they perceived it to be a clean, quick and painless method. ${ }^{19}$ The authors discussed influencing these perceptions as a possible way to prevent suicides by hanging.

The increase in popularity of the "choking game" among young people, which also involves suffocation, is a concern. The "choking game" involves depriving the brain of oxygen to

\begin{tabular}{|c|c|c|c|c|c|c|c|c|c|c|c|c|c|c|c|c|}
\hline \multirow[b]{3}{*}{ Year } & \multicolumn{4}{|c|}{ All } & \multicolumn{4}{|c|}{ Firearm } & \multicolumn{4}{|c|}{ Poisoning } & \multicolumn{4}{|c|}{ Suffocation } \\
\hline & \multicolumn{2}{|c|}{ Boys } & \multicolumn{2}{|c|}{ Girls } & \multicolumn{2}{|c|}{ Boys } & \multicolumn{2}{|c|}{ Girls } & \multicolumn{2}{|c|}{ Boys } & \multicolumn{2}{|c|}{ Girls } & \multicolumn{2}{|c|}{ Boys } & \multicolumn{2}{|c|}{ Girls } \\
\hline & $10-14$ & $15-19$ & $10-14$ & $15-19$ & $10-14$ & $15-19$ & $10-14$ & $15-19$ & $10-14$ & $15-19$ & $10-14$ & $15-19$ & $10-14$ & $15-19$ & $10-14$ & $15-19$ \\
\hline 1982 & 2.2 & 20.7 & 0.6 & 3.5 & 0.7 & 10.9 & 0.1 & 0.9 & 0.1 & 2.5 & 0.2 & 1.3 & 1.3 & 6.0 & 0.2 & 0.7 \\
\hline 1983 & 2.1 & 20.5 & 0.5 & 3.4 & 0.7 & 10.6 & 0.1 & 1.0 & 0.1 & 2.4 & 0.1 & 1.3 & 1.3 & 6.1 & 0.2 & 0.7 \\
\hline 1984 & 2.0 & 20.3 & 0.5 & 3.6 & 0.6 & 10.0 & 0.1 & 0.9 & 0.1 & 2.8 & 0.1 & 1.3 & 1.3 & 6.1 & 0.2 & 0.8 \\
\hline 1985 & 2.1 & 20.1 & 0.5 & 3.8 & 0.6 & 9.9 & 0.1 & 1.0 & 0.1 & 2.7 & 0.1 & 1.3 & 1.4 & 6.2 & 0.3 & 1.1 \\
\hline 1986 & 2.2 & 19.9 & 0.5 & 3.7 & 0.6 & 9.3 & 0.2 & 0.9 & 0.1 & 2.6 & 0.0 & 1.3 & 1.4 & 6.6 & 0.3 & 1.0 \\
\hline 1987 & 2.1 & 20.1 & 0.6 & 3.7 & 0.6 & 9.4 & 0.2 & 0.9 & 0.1 & 2.7 & 0.1 & 1.2 & 1.4 & 6.6 & 0.3 & 1.2 \\
\hline 1988 & 2.3 & 20.2 & 0.6 & 3.9 & 0.8 & 9.5 & 0.1 & 0.9 & 0.1 & 2.6 & 0.1 & 1.3 & 1.4 & 6.8 & 0.3 & 1.3 \\
\hline 1989 & 2.3 & 20.7 & 0.7 & 3.8 & 0.9 & 9.6 & 0.1 & 0.7 & 0.1 & 2.3 & 0.1 & 1.4 & 1.3 & 7.4 & 0.4 & 1.4 \\
\hline 1990 & 2.3 & 20.7 & 0.7 & 4.0 & 0.9 & 9.5 & 0.1 & 0.7 & 0.1 & 2.1 & 0.1 & 1.4 & 1.2 & 7.6 & 0.5 & 1.6 \\
\hline 1991 & 2.3 & 20.3 & 1.0 & 4.3 & 0.9 & 9.4 & 0.2 & 0.7 & 0.0 & 2.0 & 0.1 & 1.4 & 1.3 & 7.6 & 0.6 & 1.8 \\
\hline 1992 & 2.6 & 20.0 & 1.1 & 4.7 & 0.9 & 9.0 & 0.3 & 0.7 & 0.0 & 1.7 & 0.1 & 1.4 & 1.5 & 8.0 & 0.7 & 2.2 \\
\hline 1993 & 2.6 & 20.6 & 1.4 & 4.8 & 0.7 & 8.4 & 0.3 & 0.7 & 0.0 & 1.7 & 0.1 & 1.1 & 1.6 & 8.8 & 1.0 & 2.4 \\
\hline 1994 & 2.8 & 19.9 & 1.4 & 4.8 & 0.6 & 7.5 & 0.3 & 0.7 & 0.1 & 1.7 & 0.1 & 1.1 & 1.9 & 8.9 & 1.0 & 2.6 \\
\hline 1995 & 3.0 & 20.0 & 1.5 & 4.9 & 0.8 & 6.7 & 0.3 & 0.6 & 0.1 & 1.7 & 0.1 & 0.8 & 2.1 & 9.6 & 1.1 & 3.0 \\
\hline 1996 & 3.1 & 19.8 & 1.4 & 5.2 & 0.8 & 5.9 & 0.2 & 0.6 & 0.1 & 1.7 & 0.1 & 0.8 & 2.1 & 10.0 & 1.1 & 3.4 \\
\hline 1997 & 2.9 & 19.5 & 1.3 & 5.2 & 0.8 & 5.3 & 0.1 & 0.6 & 0.1 & 1.6 & 0.1 & 0.6 & 1.9 & 10.6 & 1.1 & 3.5 \\
\hline 1998 & 3.0 & 18.5 & 1.2 & 5.2 & 0.8 & 4.7 & 0.1 & 0.6 & 0.1 & 1.6 & 0.0 & 0.6 & 1.9 & 10.5 & 1.0 & 3.7 \\
\hline 1999 & 2.6 & 17.7 & 1.3 & 5.4 & 0.8 & 4.2 & 0.1 & 0.6 & 0.1 & 1.5 & 0.1 & 0.6 & 1.7 & 10.4 & 1.1 & 3.9 \\
\hline 2000 & 2.2 & 16.5 & 1.4 & 5.3 & 0.6 & 3.9 & 0.1 & 0.4 & 0.1 & 1.4 & 0.0 & 0.6 & 1.4 & 9.7 & 1.2 & 3.8 \\
\hline 2001 & 2.0 & 15.8 & 1.2 & 5.1 & 0.4 & 3.4 & 0.0 & 0.4 & 0.0 & 1.2 & 0.0 & 0.5 & 1.4 & 9.7 & 1.1 & 3.7 \\
\hline 2002 & 1.8 & 14.9 & 1.3 & 5.1 & 0.3 & 2.9 & 0.0 & 0.3 & 0.0 & 1.2 & 0.1 & 0.6 & 1.4 & 9.5 & 1.1 & 3.6 \\
\hline 2003 & 1.6 & 14.3 & 1.4 & 5.3 & 0.2 & 2.7 & 0.0 & 0.2 & 0.0 & 0.9 & 0.1 & 0.6 & 1.3 & 9.3 & 1.3 & 3.9 \\
\hline 2004 & 1.6 & 13.4 & 1.5 & 5.0 & 0.2 & 2.6 & 0.0 & 0.2 & 0.0 & 0.8 & 0.1 & 0.5 & 1.3 & 8.8 & 1.4 & 3.9 \\
\hline 2005 & 1.6 & 12.7 & 1.5 & 5.0 & 0.2 & 2.3 & 0.0 & 0.2 & 0.0 & 0.8 & 0.1 & 0.5 & 1.3 & 8.5 & 1.4 & 3.9 \\
\hline 2006 & 1.5 & 12.2 & 1.5 & 5.2 & 0.2 & 2.2 & 0.0 & 0.1 & 0.0 & 0.7 & 0.1 & 0.6 & 1.3 & 8.2 & 1.4 & 4.1 \\
\hline
\end{tabular}


induce a euphoric effect. Two distinct mechanisms are used to achieve this - strangulation (carotid artery occlusion) and self-induced hypocapnia (hyperventilation and breathholding, with or without chest compression). The "game" can turn deadly if the participant being choked is physiologically susceptible or if the pressure is not released quickly enough after the loss of consciousness. Deaths resulting from the "choking game" have the potential to be misclassified as suicides, especially when the "game" is played alone..$^{5-7}$

The prevalence and influence of the Internet and social media in the lives of young Canadians cannot be discounted in this discussion and warrants further research to understand its risks related to suicide. The term "cybersuicide" has evolved to describe the numerous websites, chat rooms and blogs promoting suicide and suicidal ideation. Such sites are obviously troubling; yet, paradoxically, the Internet and social media also hold potential benefits for the prevention of suicide. ${ }^{12-15}$

\section{Limitations}

Misclassifications of suicides as unintentional or being of undetermined intent may lead to underreporting; however, studies that have investigated this issue using American data conclude the data still reflect actual trends, but caution is advised in using the information to make international comparisons. ${ }^{20}$

We examined deaths by suffocation, poisoning and firearms that had been classified as "other intents" and saw no trends to suggest misclassification may have changed during the period of our study. Previous studies have shown that investigated deaths by nonviolent means, such as poisoning, are difficult to classify as suicides without sufficient evidence of intent (e.g., a suicide note). ${ }^{21}$

The degree to which suffocations resulting from the "choking game" may be misclassified as suicides is unknown. However, a report from the Centers for Disease Control and Prevention suggests that these deaths are not likely to account for any substantial portion of the recent increases seen in deaths by hanging or self-suffocation. ${ }^{6}$

\section{Conclusion}

Our results show that traditional patterns of suicide among children and adolescents are changing. Suicide rates among female children and adolescents are increasing, whereas those among male children and adolescents are decreasing. Limiting access to firearms, poisons and elevated structures has some potential to mitigate the risk of suicide; however, suffocation, the predominant means of committing suicide among boys and girls, is not amenable to this type of primary prevention. Additional research is required to understand the complex factors involved in this phenomenon and to develop prevention initiatives that address a changing landscape.

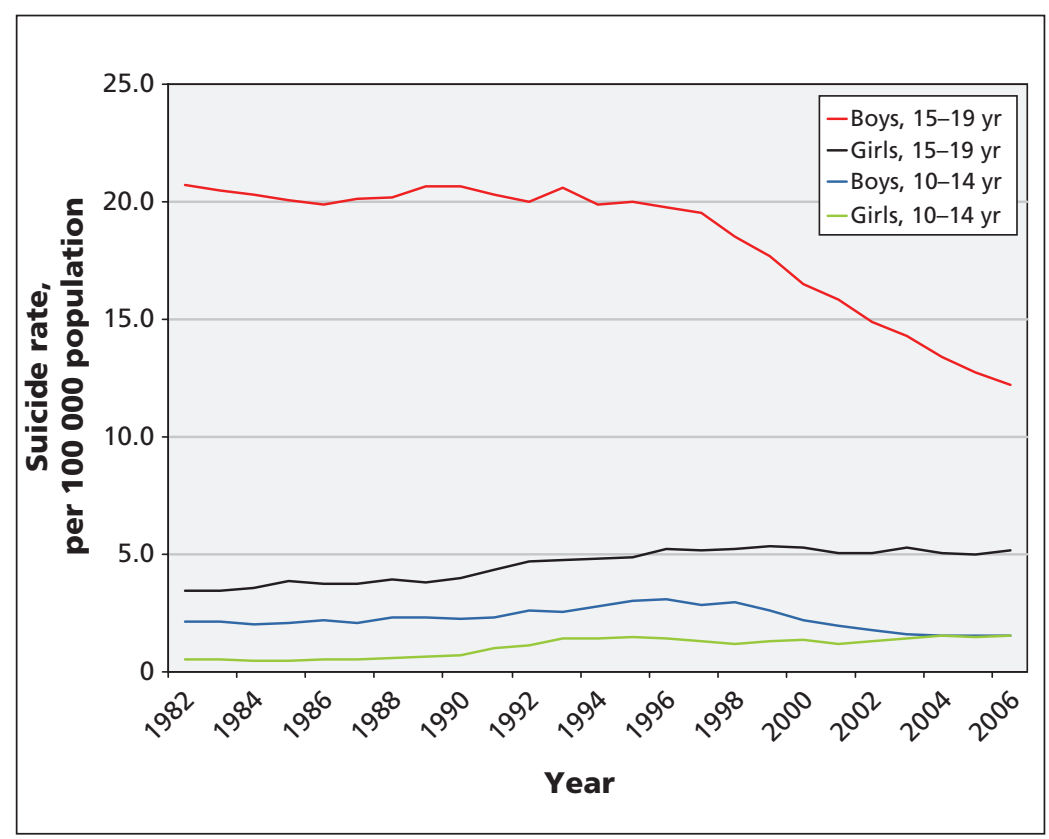

Figure 2: Overall deaths by suicide among Canadian children and adolescents by sex and age group. Rates were standardized per 100000 population* using the Canadian population in 1991 . *Calculated using a 5-point central moving average.

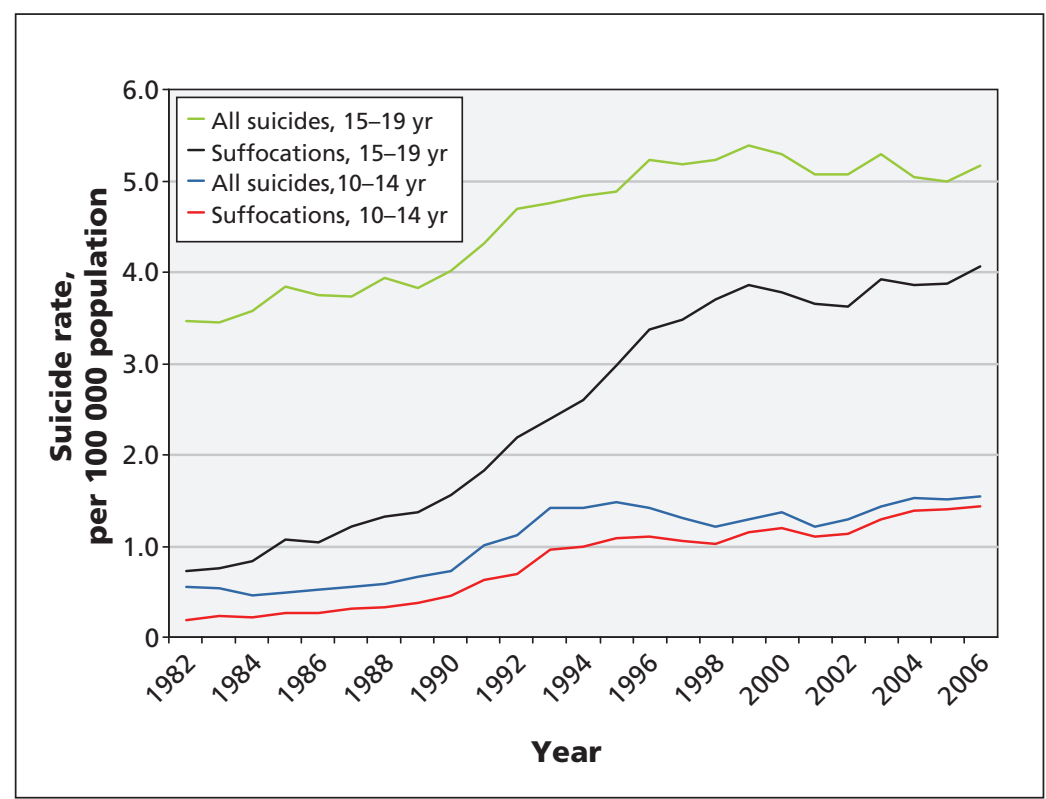

Figure 3: Deaths by suicide (overall and by suffocation) among Canadian female children and adolescents. Rates were standardized per 100000 population* using the Canadian population in 1991. *Calculated using a 5-point central moving average. 


\section{References}

1. Public Healthy Agency of Canada. Analysis of Statistics Canada mortality data. Ottawa (ON): The Agency; 2012.

2. Pan SY, Desmeules M, Morrison H, et al. Adolescent injury deaths and hospitalization in Canada: magnitude and temporal trends (1979-2003). J Adolesc Health 2007;41:84-92.

3. Pan SY, Ugnat AM, Semenciw R, et al. Trends in childhood injury mortality in Canada, 1979-2002. Inj Prev 2006;12:155-60.

4. Lubell KM, Kegler SR, Crosby AE, et al. Suicide trends among youths and young adults aged 10-24 years - United States, 1990-2004. MMWR Morb Mortal Wkly Rep 2007;56:905-8.

5. Macnab AJ, Deevska M, Gagnon F, et al. Asphyxial games or "the choking game": a potentially fatal risk behavior. Inj Prev 2009; $15: 45-9$.

6. Unintentional strangulation deaths from the "choking game" among youths aged 6-19 — United States, 1995-2007. MMWR Morb Mortal Wkly Rep 2008;57:141-4.

7. Egge MK, Berkowitz CD, Toms C, et al. The choking game: a cause of unintentional strangulation. Pediatr Emerg Care 2010; 26:206-8.

8. Posner K, Melvin GA, Stanley B, et al. Factors in the assessment of suicidality in youth. CNS Spectr 2007;12:156-62.

9. Brent DA. Firearms and suicide. Ann N Y Acad Sci 2001;932: 225-39; discussion;239-40.

10. Sinyor M, Levitt AJ. Effect of a barrier at Bloor Street viaduct on suicide rates in Toronto: natural experiment. BMJ 2010;341:c2884.

11. Centers for Disease Control and Prevention. Suicides due to alcohol and/or drug overdose: a data brief from the National Violent Death Reporting System. Atlanta (GA): The Centers; 2011. Available: www.cdc.gov/violenceprevention/pdf/NVDRS _Data_Brief-a.pdf (accessed 2011 Nov. 7).

12. Dunlop SM, More E, Romer D. Where do youth learn about suicides on the Internet, and what influence does this have on suicidal ideation? J Child Psychol Psychiatry 2011;52:1073-80.

13. Alao AO, Soderberg M, Pohl EL, et al. Cybersuicide: review of the role of the internet on suicide. Cyberpsychol Behav 2006;9:489-93.

14. Ruder TD, Hatch GM, Ampanozi G, et al. Suicide announcement on facebook. Crisis 2011;32:280-2.
15. Birbal R, Maharajh HD, Birbal R, et al. Cybersuicide and the adolescent population: Challenges of the future? Int J Adolesc Med Health 2009;21:151-9.

16. Anderson RN, Miniño AM, Hoyert DL, et al. Comparability of cause of death between ICD-9 and ICD-10: preliminary estimates. Natl Vital Stat Rep 2001;49:1-32.

17. Geran L, Tully P, Wood P, et al. Comparability of ICD-10 and ICD-9 for mortality statistics in Canada. Ottawa (ON): Statistics Canada; 2005. Available: www.statcan.gc.ca/pub/84-548-x/84548-x2005001-eng.htm (accessed 2011 Oct. 31).

18. Karjalainen A. International statistical classification of diseases and related health problems (ICD-10) in occupational health. 10th rev. Geneva (Switzerland): The Organization; 2004.

19. Biddle L, Donovan J, Owen-Smith A, et al. Factors influencing the decision to use hanging as a method of suicide: qualitative study. Br J Psychiatry 2010;197:320-5.

20. Rockett IR, Kapusta ND, Bhandari R. Suicide misclassification in an international context: revisitation and update. Suicidology Online 2011;2:48-61.

21. Donaldson AE, Larsen GY, Fullerton-Gleason L, et al. Classifying undetermined poisoning deaths. Inj Prev 2006;12:338-43.

Affiliations: From the Injury Section, Health Surveillance and Epidemiology Division, Centre for Chronic Disease Prevention and Control, Public Health Agency of Canada, Ottawa, Ont.

Contributors: Robin Skinner drafted the manuscript and both authors contributed to its revision. Both authors worked in partnership on the development of the methods, and the analysis and interpretation of the data. Robin Skinner takes responsibility for the paper as a whole. Both authors approved the final version submitted for publication.

Acknowledgements: The authors thank Sharon Bartholomew, Mitsi Cardinal, Minh T. Do, Margaret Herbert, Wendy Thompson and Anne-Marie Ugnat for their review of this manuscript. 